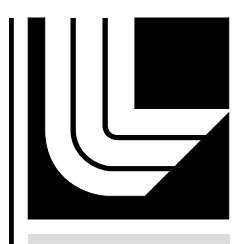

LAWRENCE LIVERM ORE N A TIO NAL LABORATORY

\title{
Contribution to Fusion Materials Semiannual Report
}

J. Marian, W. Meier

February 27, 2012 
This document was prepared as an account of work sponsored by an agency of the United States government. Neither the United States government nor Lawrence Livermore National Security, LLC, nor any of their employees makes any warranty, expressed or implied, or assumes any legal liability or responsibility for the accuracy, completeness, or usefulness of any information, apparatus, product, or process disclosed, or represents that its use would not infringe privately owned rights. Reference herein to any specific commercial product, process, or service by trade name, trademark, manufacturer, or otherwise does not necessarily constitute or imply its endorsement, recommendation, or favoring by the United States government or Lawrence Livermore National Security, LLC. The views and opinions of authors expressed herein do not necessarily state or reflect those of the United States government or Lawrence Livermore National Security, LLC, and shall not be used for advertising or product endorsement purposes.

This work performed under the auspices of the U.S. Department of Energy by Lawrence Livermore National Laboratory under Contract DE-AC52-07NA27344. 


\section{Contribution to Fusion Materials Semiannual Report}

Jaime Marian and Wayne Meier

Lawrence Livermore National Laboratory

\section{Objectives}

The objectives of this work are the following:

- $\quad$ The application of micro and mesoscale modeling techniques to study dislocation properties in ferritic and W-based materials.

- The development of computational models and tools to study damage accumulation in $>1 \mathrm{dpa}$ (fusion-like) conditions, both for Fe and W-based alloys.

\section{Background and approaches used}

\section{Plasticity of Irradiated Ferritic Alloys}

The high-temperature strength of structural ferritic alloys (ferritic/martensitic steels, ODS steels, bcc refractory alloys) hinges on the thermal stability of second phase particles and their interactions with dislocations. Irradiation damage can modify the structure and stability of both the particles and dislocations, particularly by the introduction of gas atoms, point defects and point defect clusters. The three aspects of materials strength that we are studying are:

a) Computation of dislocation mobility functions (stress-velocity relations) as a function of temperature and dislocation character. This will be done via molecular dynamics (MD) simulations of single dislocation motion under applied shear stress. This is a fundamental input to dislocation dynamics (DD) simulations and also provides fundamental insights into the high-temperature plastic behavior of ferritic materials.

b) Simulations of dislocation-obstacle interactions using MD and DD. This subtask includes simulating the effect on dislocation glide of precipitates (e.g., $\alpha^{\prime} \mathrm{Cr}$ precipitates), ODS particles, and irradiation induced defect clusters (e.g. voids, dislocation loops, etc.).

c) Implementation of this information (dislocation mobilities and dislocation-defect interaction rules) into DD codes that will allow us to study plasticity of single crystals Fe alloys under relevant irradiation conditions.

\section{Accelerated Stochastic Techniques for Radiation Damage Kinetics}

The use of standard kinetic Monte Carlo ( $\mathrm{kMC}$ ) for radiation damage calculations suffers from a number of limitations that prevent it from accessing the fluences (doses) relevant for fast and fusion reactors. Among them, the stiffness of the rate spectrum, i.e. a large disparity in the rate constants of the evolving species, is one of the most noteworthy. Also, due to its intrinsic time discreticity, the 'next' time step is impossible to predict, which makes parallelization a difficult task. We have developed a method that circumvents this difficulty by using automatic time synchronization based on the use of 'null' events. The algorithm, termed spkMC (synchronous parallel kMC), has now been extended to discrete lattices with good success. At present, we have done only calculations in simple cubic Ising systems but the idea is to start binary alloys in the next fiscal year to study phenomena such as radiation-enhanced diffusion/segregation.

In addition, another line of work that being pursued within the base program is the development of stochastic cluster dynamics based on the Gillespie method. In contrast to providing existence probabilities for all possible species in an irradiated volume, as mean-field rate theory, this method samples from the cumulative probability distribution of the species existing at a given instant, adding new species as required by the kinetics of the system. In this fashion, the method introduces some natural stochastic 
variability to cluster dynamics calculations. Additionally, the method facilitates the treatment of multidimensional species such as vacancy-He complexes or mixed interstitial dumbbells. These two tasks build on well-known and thoroughly tested and applied computational techniques, but that had recently shown signs of saturation in terms of computational power. We have demonstrated the capabilities of the method by carrying out calculations of triple dpa/He/H irradiations in model $\mathrm{FeCr}$ alloys up to $50 \mathrm{dpa}$. Next, we will introduce chemical effects in the form of passivation of void surfaces by $\mathrm{H}$ atoms and $\mathrm{H}-\mathrm{He}$ synergisms to study the effect of simultaneous vs. sequential implantation on swelling in these materials.

\section{Technical Progress}

In the period covered by this report, we have calculated the mobilities of screw and edge dislocations in $\mathrm{Fe}$, both of which have been published in Physical Review B. The stochastic cluster dynamics method was published in Journal of Nuclear Materials, as well as the calculation of the fraction of He that ends up in substitutional sites as part of its own collisional process. This is an important value in view of the extreme differences in mobility between substitutional and interstitial sites.

Also, we have tested five interatomic potentials for $\mathrm{W}$ and assessed their feasibility for screw dislocation simulations. We have identified two potentials that are superior to the rest for this kind of simulations. These two are advantageous because they predict the correct transition barriers for dislocation motion as given by electronic structure calculations. The findings of this work have been submitted to Physical Review B. A short but relevant study on the application of boundary conditions for MD simulations has also been performed and submitted for publication in Modelling and Simulation in Materials Science and Engineering.

\section{Calculation of edge dislocation mobilities in bcc Fe}

In the traditional picture of plasticity in bcc metals, edge dislocations have been assumed to play a minor role due to their high mobility with respect to screw dislocations, which then control plastic flow. $1 / 2<111>\{110\}$ edge dislocations indeed fit this description, as it has been shown by way of numerous atomistic simulations. However, $1 / 2<111>\{112\}$-edge dislocations have been comparatively much less studied. The recent discovery of a possible regime where they move slowly via thermally activated kinkpair nucleation may have implications in the plastic behavior of bcc materials. Because dislocation mobilities are very difficult to measure experimentally, we are calculating comprehensive mobility laws for both types of edge dislocations as a function of temperature and stress using molecular dynamics simulations. Our results confirm the existence of clearly delimited thermally activated and phonon drag dynamic regimes for $1 / 2<111>\{112\}$ edge dislocations and of a single viscous drag regime for their $1 / 2<111>\{110\}$ counterparts. We also provide an analysis to relate the difference in mobility to the dislocation core properties. Our fitted mobility laws may be used in dislocation dynamics simulations of plastic flow involving millions of segments. 

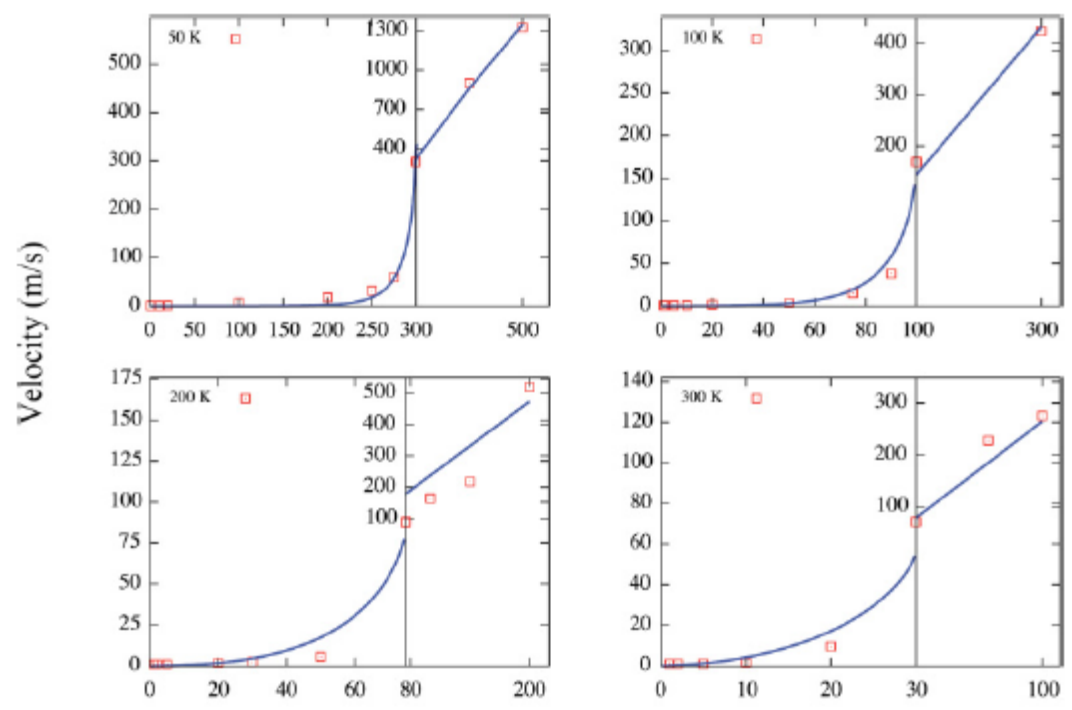

Applied stress (MPa)

Fig. $1.1 / 2<111>\{112\}$ edge dislocation velocities as a function of stress and temperature.

Dislocation velocities as a function of stress and temperature are known as mobility functions. The methodology to compute dislocation mobilities using MD simulations has been explained in detail elsewhere [8], but basically consists of generating a dislocation in a simulation box oriented along the line, glide, and plane normal directions (which, in turn, are dictated by the crystallography. Figure 1 shows results of $1 / 2<111>\{112\}$ edge dislocation velocities as a function of temperature and stress. The mobility function that best fits these data is:

$$
v_{(112)}=1.68 \times 10^{4} \frac{\tau_{n}(T)}{T} \exp \left\{-0.15\left[1-\tau_{n}(T)^{0.13}\right]^{0.68}\right\}
$$

where $T$ is the temperature and $T_{n}$ is a temperature normalized stress that marks the transition from thermally activated motion to phonon drag.

To obtain the above equation it was necessary to calculate the energy of a double kink on a $1 / 2<111>\{112\}$-edge dislocation. The structure of the kinks can be seen in Figure 2. A value of 0.15 for the double kink was obtained. This parameter goes into the fitting of the mobility law. 


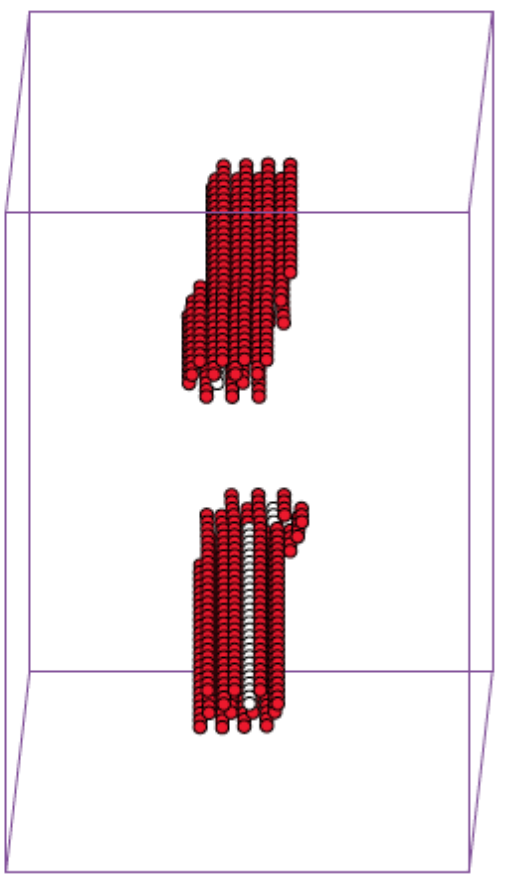

Fig. 2: Atomistic structure of two single kinks in a $1 / 2<111>\{112\}$ edge dislocation. The energy of one kink is $0.075 \mathrm{MeV}$. 


\section{Development of the stochastic cluster dynamics methodology}

Mean field rate theory (MFRT) has been the workhorse method for simulations of radiation damage accumulation for over 40 years. As its name indicates, MFRT owes its high computational efficiency to the mean-field approximation, in which the details concerning the spatial distribution of defects are assumed unimportant. MFRT models evolve in time by solving forward in time a set of ODEs for defects and defect cluster concentrations.

While efficient for simple material models, MFRT struggles to describe damage accumulation in realistically complex materials where it suffers from combinatorial explosion in the number of ODEs that must be solved. Simply put, rate theory calculations are prohibitively expensive for multidimensional cluster spaces. Our work here is to recast the MFRT method in the form of a stochastic cluster dynamics (SCD) algorithm following the approach proposed earlier by Gillespie in the context of bio-chemical reaction networks [6]. In SCD, rather than solving ODEs for defect cluster concentrations in an infinite material volume, integer-valued defect populations evolve stochastically in a finite material volume, one defect reaction (event) at a time. Thus, only those defects and defect clusters that are actually present in the simulation volume are considered. This circumvents combinatorial explosion allowing SCD simulations of complex defect populations defined in cluster spaces of arbitrary dimensionality. This newly gained ability of SCD to model complex defect populations is essential for our project. We intend to rely on the SCD method for simulations of irradiated materials involving multiple defect and chemical species.

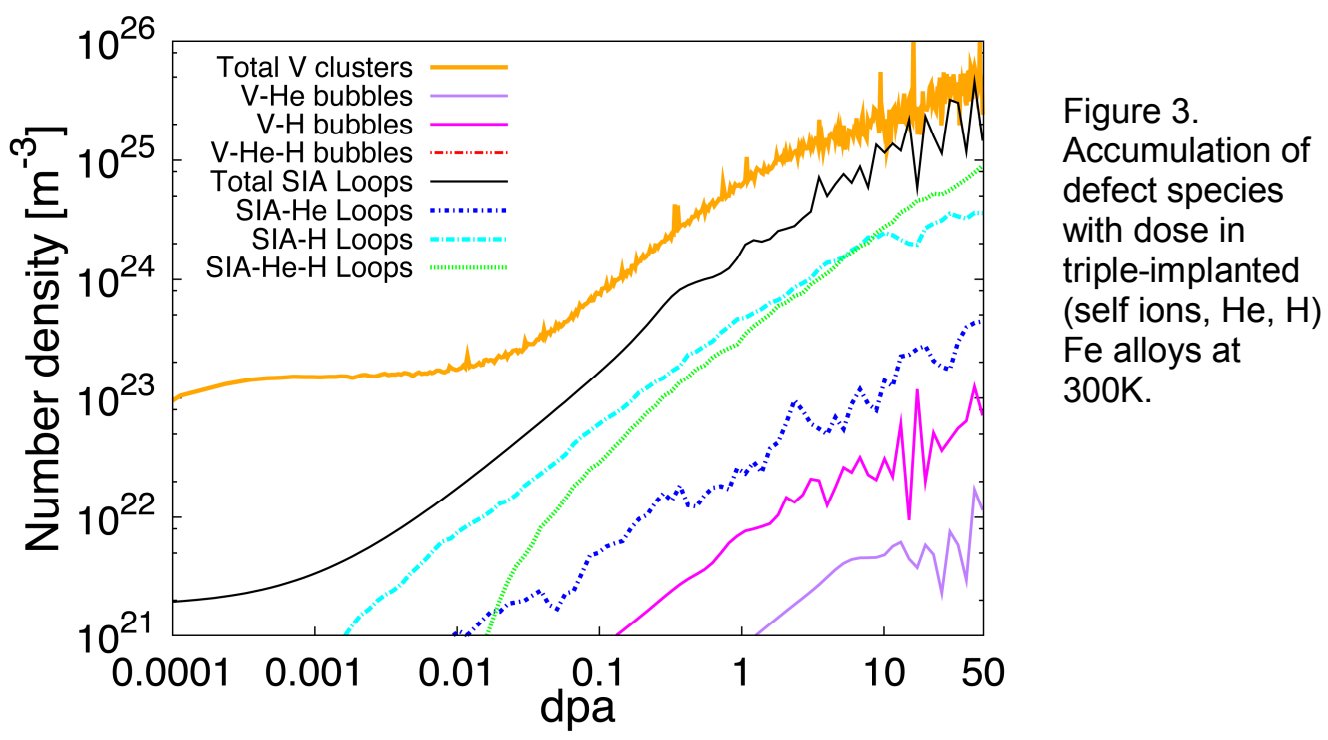

In conditions where spatial correlations and fluctuations in spatial distribution of defects are deemed important, (particle-based) object kinetic Monte Carlo (OKMC) is the method of choice. However, standard OKMC simulations can reach dose limit of the order of $0.01 \mathrm{dpa}$. By contrast, SCD enables simulations up to tens of dpa. As a demonstration of the method capabilities, Figure 3 shows a recent calculation of defect accumulation up to doses of 50 dpa in tri-implanted $\mathrm{FeCr}$ alloys. 


\section{Study of the effect of the interatomic potential on dislocation motion in W}

As mentioned in $16 \mathrm{~b}, \mathrm{~W}$ is one of the main candidates for plasma-facing applications in magnetic fusion reactors. W, however, is very brittle and efforts are being conducted to find more ductile W alloys suitable for nuclear environments. In this sense, we are developing interatomic potentials for binary $\mathrm{W}$ systems aimed at obtaining ductile alloys from an atomistic perspective. Before these efforts are underway, we need to characterize pure W potentials properly. W has comparatively been less studied than other bcc metals at the atomistic level and thus much work is still required to come up with reliable atomistic models. In a certain sense, because the plastic behavior of bcc metals like $\mathrm{W}$ is governed by screw dislocations motion, ductility equals higher dislocation mobility. Here we compare five different interatomic potentials for $\mathrm{W}$ and calculate dislocation motion in a wide range of temperatures.

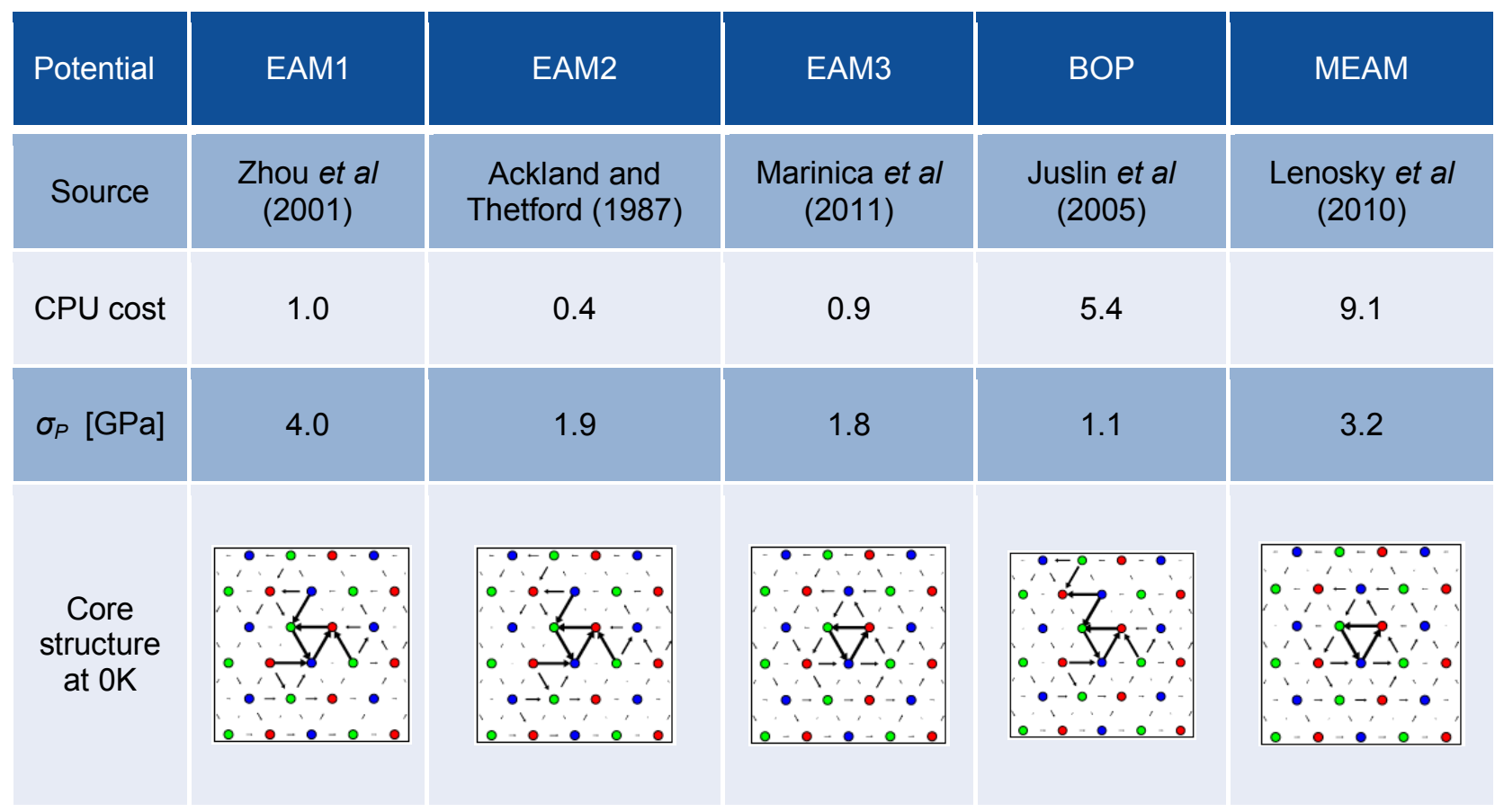

Table I: Comparison of static properties of semiempirical interatomic potentials for W.

The potentials tested include three EAM (embedded atom method), one BOP (bond order potential) and one modified EAM (MEAM). Table I describes the basic features predicted by each potential at OK. On the basis of electronic structure calculations, which predict a compact core and a Peierls stress $\sigma_{P}$ of 2.8 GPa, one can filter out potentials EAM1, EAM2 and BOP.

In addition we have calculated the Peierls barrier and the gamma surface for all potentials. The Peierls barrier is given in Fig 4 along with some DFT data for reference. Clearly, the MEAM and EAM3 potentials come closest to the shape and value of the DFT results. 


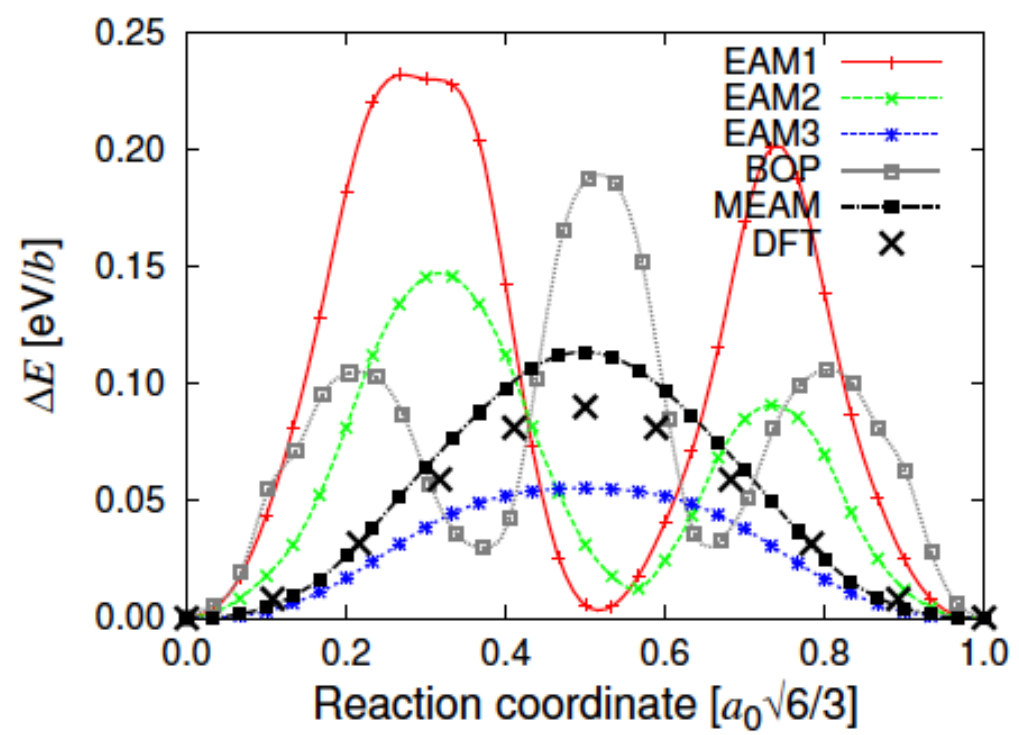

Fig. 4. Peierls trajectory for all five $\mathrm{W}$ potentials tested. DFT results are shown for comparison.

Fig 5 shows the corresponding gamma surface. Again the EAM3 and MEAM do best in reproducing the DFT data.

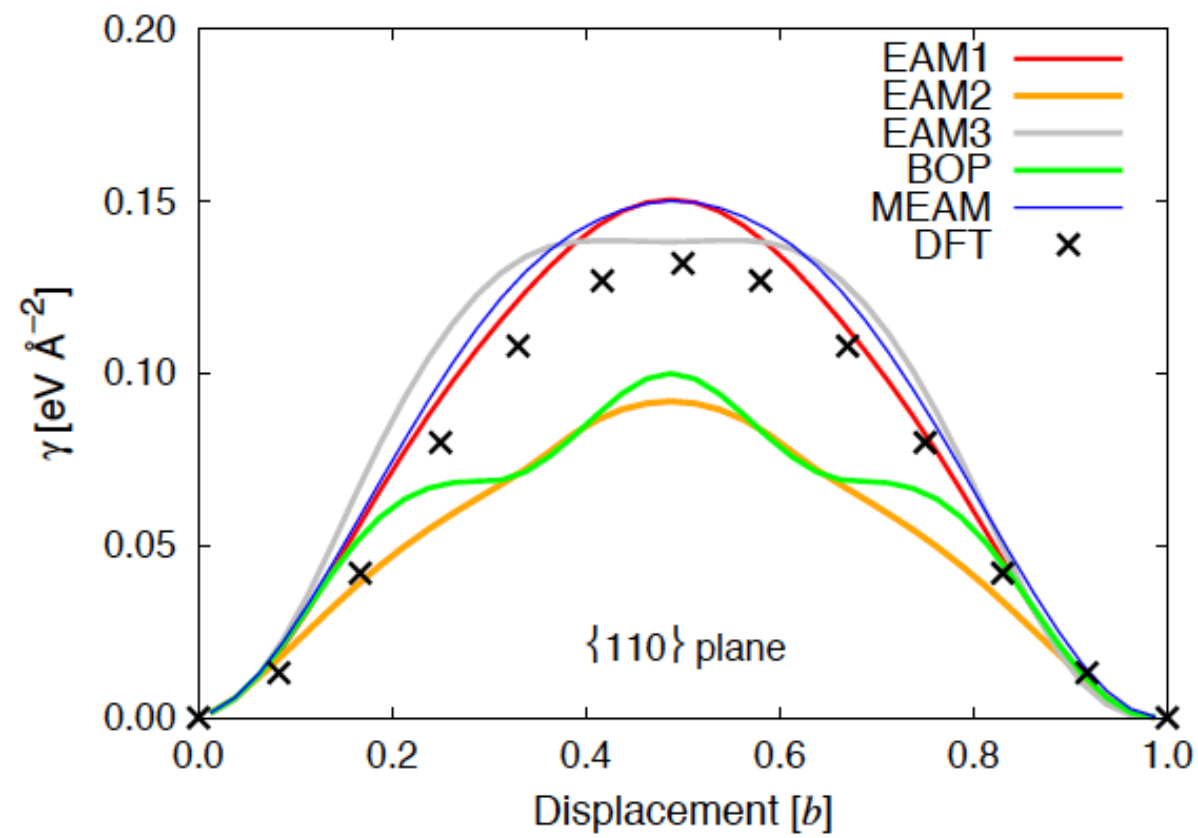

Fig. 5. Gamma surface for all five W potentials tested. DFT results are shown for comparison. 


\section{Publications}

S. Queyreau, J. Marian, M. R. Gilbert, and B. D. Wirth, "Edge dislocation mobilities in bcc Fe obtained by molecular dynamics", Physical Review B 84 (2011) 064106.

J. Marian and V. V. Bulatov, "Stochastic cluster dynamics method for simulations of multispecies irradiation damage accumulation, Journal of Nuclear Materials 415 (2011) 84.

Paul Erhart and Jaime Marian, "Calculation of the substitutional fraction of ion-implanted $\mathrm{He}$ in an $\alpha-\mathrm{Fe}$ target", Journal of Nuclear Materials 414 (2011) 426.

D. Cereceda, J. M. Perlado and J. Marian, "The effect of boundary conditions on stress-controlled molecular dynamics simulations of dislocation motion in tungsten", submitted to Modelling and Simulation in Materials Science and Engineering (2012).

D. Cereceda, J. M. Perlado, S. Queyreau, A. Stukowski, M.-C. Marinica, L. Ventelon and J. Marian, "Temperature induced screw dislocation core transformation and its effect on mobility in pure W", submitted to Physical Review B (2012).

\section{References}

[1] C.J. Ortiz and M.J. Caturla, J. Comp-Aided Mater Des 14 (2007) 171.

[2] Caturla, M.J., Soneda, N., Alonso, E., Wirth, B.D., Díaz de la Rubia, T. and Perlado, J.M., J. Nucl. Mater. 276 (2000) 13.

[3] SI Golubov, RE Stoller, SJ Zinkle, et al., Journal of Nuclear Materials 361 (2007) 149.

[4] RE Stoller, SI Golubov, C Domain, et al., Journal of Nuclear Materials 382 (2008) 77.

[5] E Martínez, J Marian, MH Kalos and JM Perlado, Journal of Computational Physics 227 (2008) 3804.

[6] DT Gillespie, The Journal of Physical Chemistry 81 (1977) 2340-2361.

[7] VV Bulatov et al., Nature 440 (2006) 1174-1178.

[8] J Marian and A Caro, Physical Review B 74 (2006) 024113.

[9] E Martinez, PR Monasterio and J Marian, Journal of Computational Physics 230 (2011) 1359.

[10] E Martinez, J Marian, MH Kalos, JM Perlado, Journal of Computational Physics 227 (2008) 3804.

[11] L Romaner, C Ambroschl-Draxl and R Pippan, PRL 104 (2010) 195503.

[12] X Tian and C Woo, Materials Science \& Engineering A 369 (2004) 210.

[13] T Ahlgren, K Heinola, N Juslin and A Kuronen, Journal of Applied Physics 107 (2010) 033516.

[14] X. W. Zhou and et al., Acta Materialia 49, 4005 (2001).

[15] G. J. Ackland and R. Thetford, Philosophical Magazine A 56, 15 (1987).

[16] M.-C. Marinica and et al., (2012) to be submitted.

[17] N. Juslin, P. Erhart, P. Traskelin, J. Nord, K. O. E. Henriksson, K. Nordlund, E. Salonen, and K. Albe, J. Appl. Phys. 98, 123520 (2005).

[18] T. J. Lenosky, B. Sadigh, E. Alonso, V. V. Bulatov, T. D. de la Rubia, J. Kim, A. F. Voter, and J. D. Kress, Modeling Simul. Mater. Sci. Eng. 8, 825 (2000) 